\title{
Essais de performance des indicateurs et intégrateur de température pour la chaine du froid : méthodologie de la norme NF E 18-100 et résultats
}

\author{
Eric DEVIN $^{(1)}$, Gérald CAVALIER ${ }^{(2)}$, Sandrine LECOCQ ${ }^{(2)}$ \\ ${ }^{(1)}$ (2) Cemafroid SAS - 5 avenue des Prés - 94266 Fresnes Cedex
}

\begin{abstract}
Temperature indicators (TI) and time-temperature integrators (TTI) are more and more used to control and assess cold chain particularly, in food, health and chemistry sectors. These tools are used to monitor temperature-sensitive products. The time/temperature indicator is used to indicate, by a visible change of state, an overlap of a temperature limit, instantly or for a given period. It allows the consumption of a capital time/temperature set to be indicated by a visible change of state. The time-temperature indicator is easy to use, cheap and understandable by everybody.
\end{abstract}

\begin{abstract}
Mots clés
IT ITT temperature indicator indicateur integrator intérateur MKT TCM excursion threshold

\section{1 - Introduction}

Le développement assez récent de dispositifs détectant les excursions de température (parfois en les intégrant en fonction du temps) pour faciliter les études de risques menées par les pharmaciens, procure de nouveaux outils pour gérer la chaine du froid des produits de santé thermosensibles. Ces dispositifs de technologies très différentes sont des alliés de taille pour sécuriser la chaine du froid sous réserve de connaitre précisément leurs caractéristiques métrologiques. Ceci était jusqu'à présent assez difficile en raison de l'absence de norme d'essais. La norme NF 18150 publiée en 2013 fournit désormais un protocole de test adapté pour quantifier l'apport des indicateurs de température (IT) et des intégrateurs temps température (ITT) indépendamment de leur technologie. Elle aboutit à une classification (classe d'exactitude) qui permet un usage généralisé de ces techniques en assurant la traçabilité métrologique des seuils détectés.
\end{abstract}

2 - Les indicateurs de température et les intégrateurs tempstempérature : le principe

Il est possible de définir comme « indicateur de température IT » un outil qui permet d'indiquer un dépassement instantané ou pendant une durée donnée (fractionnée ou non) d'une température fixée. L'indication est réalisée par un changement visible d'état comme par exemple la couleur.
A la différence, les « intégrateurs temps-température ITT » sont des outils qui permettent d'indiquer l'atteinte d'un cumul maximum «temps/température». L'indication est aussi réalisée par un changement visible d'état. Derrière ce cumul temps-température, l'intégrateur signale le dépassement d'un critère assimilable à un quota d'énergie caractérisant l'excursion de température et susceptible de fournir une information sur la limitation de la durée de péremption d'un produit pharmaceutique. Cela implique que la loi de dégradation du produit est connue.

Techniquement, on constate donc que les IT et les ITT disposent de deux types d'indications très différentes dont l'usage est distinct :

- $\quad$ Pour les IT : Avertir le pharmacien lorsque qu'une limite haute ou basse de température (seuil) est franchie lors du stockage ou du transport d'un produit ;

- Pour les ITT: Avertir le pharmacien lorsqu'une excursion durable atteint un cumul temps température fixé. Le cumul seuil pour être utile doit être en lien avec le domaine de connaissance du produit afin d'être utilisé de manière profitable lors des études de risque en cas de non-respect de la chaîne du froid.

\section{2-1 Bases du comportement des produits thermosensibles}

L'intérêt d'utiliser des IT ou des ITT dépend avant tout de la connaissance du comportement des produits thermosensibles et de l'effet de la température sur ces produits. Le management de la chaine du froid en utilisant ces indicateurs ou intégrateurs ne peuvent donc être dissociées des résultats des études de stabilité des produits concernés standardisés par les tests ICH. En effet, les 
études de stabilité réalisées selon les tests ICH fournissent un domaine de connaissance des produits notamment :

- L'effet des basses températures $\left(<2{ }^{\circ} \mathrm{C}\right)$ et le risque de congélation ou destruction mécanique avec comme conséquence la destruction totale des produits ;

- L'effet des dépassements des températures maximales autorisées $\left(>8^{\circ} \mathrm{C}\right)$ sur la dégradation des principes actifs ayant un impact sur la date de péremption établie lors des études de stabilité.

Sur la base du domaine de connaissance des produits, il est donc possible d'utiliser les IT et ITT pour contrôler l'impact des excursions de température et pour faciliter les études de risque menées par les pharmaciens.

Ainsi, les IT seront propices à traquer des dépassements soudains de température notamment les risques de congélation ou les effets destructifs. La détection de seuil supposée instantanée permet sur toute la chaine logistique de s'assurer de l'absence ou de la présente d'un accident.

D'un autre côté, les intégrateurs temps-température permettent d'enregistrer un cumul temps température assimilable à un cumul énergétique très utile si l'on a recours à une modélisation se basant sur un processus thermo-activé répondant à la loi d'Arrhenius par exemple (usage de la température cinétique moyenne TCM).

\subsection{Excursion ayant un effet cumulatif sur la qualité du produit}

L'intérêt principal de suivre le cumul temps-température vient du fait que le produit temps température est assimilable à une énergie. Ainsi à une valeur d'énergie donnée correspondent plusieurs profils de température pour plusieurs durées d'excursion possibles. Si la dégradation d'un produit est uniquement fonction de cette énergie et non de la valeur maximale de la température, le suivi de ce cumul permet de conclure que pour ces différents profils l'impact sur le produit sera le même.

Il s'agit d'hypothèses assez fortes mais beaucoup de phénomènes chimiques sont uniquement fonction de cette énergie. Mieux, Arrhenius a pu établir une loi empirique qui relie la cinétique de ces réactions au couple temps température pour de nombreuses applications. Il a pu démonter qu'au-delà d'un seuil énergétique appelé énergie d'activation [figure 1], les apports d'énergie supplémentaires contribuent à la cinétique de la réaction, dans notre cas, la réaction de dégradation du produit. Plus la température est élevée, plus la réaction est rapide. Il en découle que si un produit est le siège d'une dégradation uniquement basée sur une loi de type Arrhenius alors, il suffit de connaitre son énergie d'activation pour connaitre l'effet d'une excursion de température.

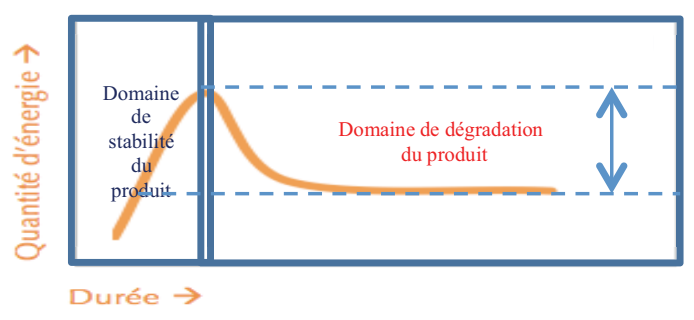

[Figure 1] illustration du phénomène d'énergie d'activation
Les pharmaciens utilisent parfois ce concept au travers d'un autre paramètre appelé température cinétique moyenne (TCM ou MKT en anglais) qui correspond à la température à laquelle un produit devrait se trouver pour une durée connue pour subir la même dégradation qu'un profil complexe de température variable.

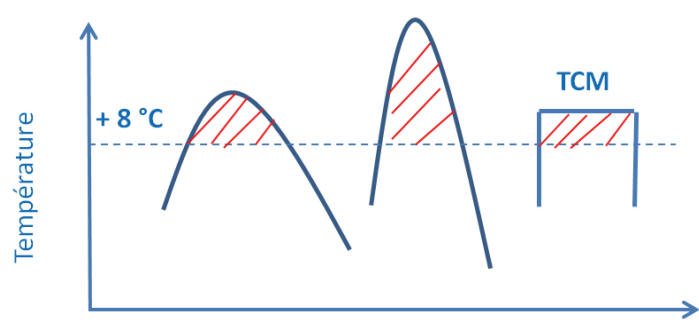

[Figure 2] Illustration de différents profils d'excursion de température correspondant à la même température cinétique moyenne (TCM)

Si l'on applique ce concept à la pharmacie, la TCM permet d'intercomparer des excursions de formes multiples [figure 2] et de conclure qu'elles sont du point de vue énergétique toutes équivalentes. Si l'énergie est le seul paramètre qui gouverne la dégradation des produits, alors, on peut conclure que la dégradation sera la même pour tous les profils correspondant à la même TCM quel que soit l'intensité de l'excursion.

Les ITT sont dans ce domaine des intégrateurs d'énergie, leur seuil étant défini par une TCM à ne pas dépasser.

\section{Les technologies d'IT et ITT}

Il existe une multitude de technologie d'indicateurs ou d'intégrateur qui se base sur deux principes généraux: Soit la mesure directe d'une température et éventuellement d'un temps. Un calcul permet de faire le lien à des seuils prédéfinis. Soit une détection d'un seuil par le biais d'une réaction physique, chimique ou biologique qui se produit sous l'effet de la température. Nous allons passer en revue ces différentes technologies.

\subsection{Les indicateurs électroniques}

Les indicateurs électroniques sont constitués d'un circuit alimenté par une batterie, d'une sonde de température généralement constituée d'une thermistance et d'un dispositif d'affichage. Si les outils du marché sont encore souvent assez encombrants avec des épaisseurs de plusieurs millimètres, des versions en développement permettent d'espérer de disposer d'ici quelques temps d'outils imprimés sur des films de l'ordre du dixième de millimètre. 

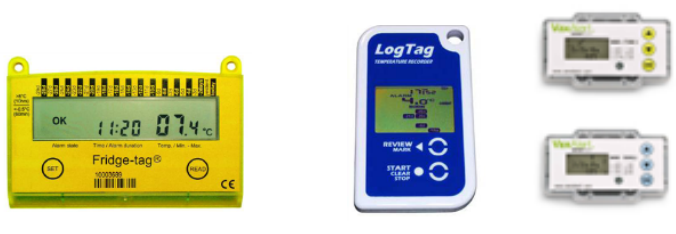

[Figure 3] Intégrateurs électroniques avec affichage du temps de dépassement de la température.

\subsection{Les indicateurs mécaniques ou physiques}

Ces indicateurs physiques utilisent les propriétés thermo-physiques d'un matériau comme sa variation de volume ou de forme en fonction de la température ou encore la rupture mécanique d'un contenant sous l'effet de la congélation d'un liquide ou le changement de position d'un bilame. Ils sont en général utilisés pour enregistrer des effets de seuil ponctuels (IT). Certains de ces indicateurs fonctionnement de manière irréversible. Ils peuvent nécessiter une procédure préalable d'activation qui complexifie leur utilisation à grande échelle.
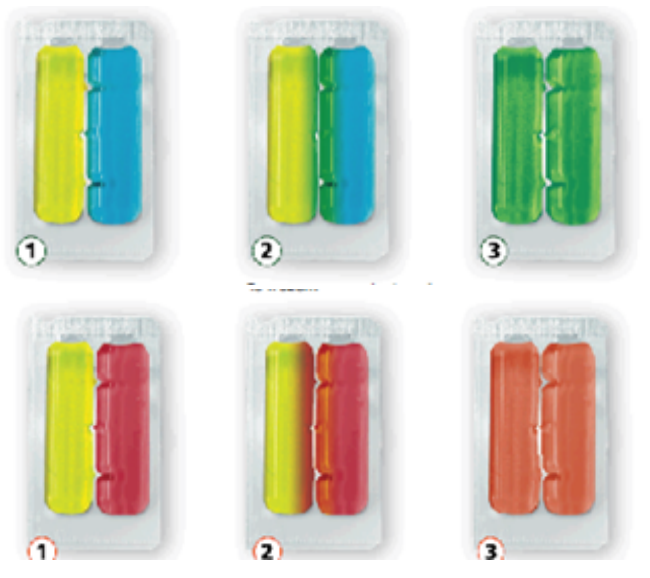

[Figure 4.] Indicateur mécanique

\subsection{Indicateurs et intégrateurs chimiques}

Les indicateurs chimiques reposent sur les propriétés thermochimiques de réactifs comme le changement de couleur d'une encre thermo-chrome en fonction de la température ou la diffusion par absorption d'un liquide sur un buvard. Ils peuvent indiquer des dépassements ponctuels (IT) ou successifs de seuil, et constituer alors des intégrateurs temps température (ITT). Le choix des réactifs ou des supports permettent de paramétrer les caractéristiques de l'ITT en fonction des types de produits concernés par l'application. Là encore, à usage unique, les phénomènes rencontrés sont irréversibles et permanents.

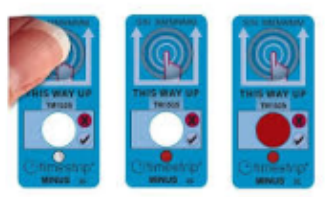

[Figure 5.] Indicateur chimique avec changement de couleur lors du dépassement. microbiologiques

Les indicateurs biologiques ou microbiologiques utilisent les propriétés thermosensibles de certains microorganismes, par exemple leur vitesse de multiplication. Ces indicateurs sont des intégrateurs (ITT). Ils utilisent également les propriétés chimiques de certaines composées issues de la multiplication des microorganismes pour induire des changements de couleur, par exemple, du fait d'un changement de PH. En fonction de la conception, ils peuvent être paramétrables.

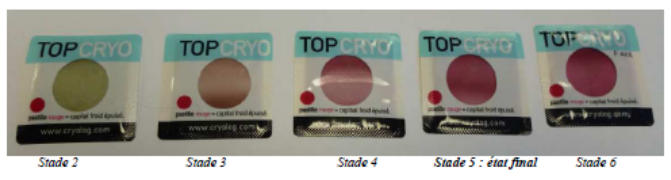

[Figure 6.] Intégrateurs microbiologiques indiquent la consommation d'un capital temps/température fixé par un changement de couleur.

C'est ce type d'intégrateur temps température qui a été étudié pour l'application de la norme E 18-100 présentée dans cet article.

4 Comment s'assurer des performances métrologiques des IT et ITT

\section{1 - la norme $\mathrm{E}$ 18-100}

L'usage des IT et ITT implique de connaitre leurs caractéristiques métrologiques pour s'assurer d'une détection correcte des seuils de température pour les IT et des seuils temps température pour les ITT. La difficulté réside dans le fait qu'un étalonnage est impossible pour les raisons suivantes :

- $\quad$ Les IT et ITT ne fournissent pas tous une mesure directe du paramètre mesuré et se limitent à une indication de franchissement ou non de ces seuils par un changement d'état comme un changement de couleur;

- Ces dispositifs ont un fonctionnement irréversible, une fois le seuil atteint, il n'est plus possible d'utiliser ces dispositifs.

Partant de ces principes, les fabricants, les utilisateurs et les experts français des indicateurs et intégrateurs temps-température se sont réunis pour élaborer une méthode de qualification et fixer les exigences minimales requises. Ces travaux ont abouti à la publication en 2013 d'une norme d'essai : la norme NF 18-100.

La norme NF 18-100 définie les méthodes d'essai pour évaluer les performances des IT et des ITT :

- Les essais les plus important sont bien évidemment les essais « d'exactitude » réalisés à température constante ou pour un profil de température dynamique; Le principe de ces essais est d'appliquer des seuils de température croissant autour de la température de référence de détection de l'indicateur pour constater la justesse et le fonctionnement correct de ce dernier [Figure 7.]. 


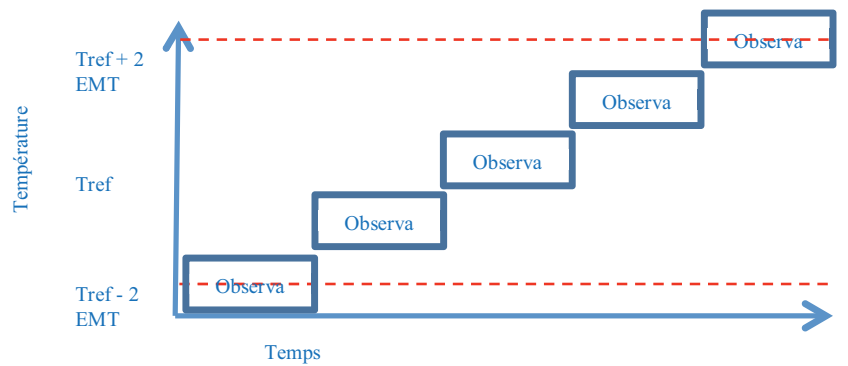

[Figure 7.] Essais d'exactitude d'un indicateur dont l'effet de seuil déclaré se trouve à Tref

La norme fixe des erreurs maximales tolérées applicables aux résultats d'essais :

- Les temps seuils réels et les températures seuils réelles sont conformes si l'écart aux temps seuils théoriques et aux températures seuil théoriques est $<10 \%$

- Pour les intégrateurs temps/température : Une durée réelle est conforme si l'écart à la durée théorique est $<10 \%$.

La norme définit aussi les modalités d'expression des résultats, de classification de l'IT ou l'ITT et de déclaration de conformité. Pour un modèle testé, on calcule ainsi pour chaque essai, la moyenne et l'écart-type des écarts aux durées théoriques (pour les indicateurs), et aux temps seuils et températures seuils théoriques (pour les intégrateurs temps/températures). Parmi les moyennes, la plus grande est retenue et permet de déterminer la classe du dispositif suivant le tableau ci-dessous :

\begin{tabular}{|c|c|c|c|c|}
\hline Classe & A & B & C & $\begin{array}{c}\text { Hors } \\
\text { classe }\end{array}$ \\
\hline $\begin{array}{c}\text { Ecart } \\
\text { moyen (\%) }\end{array}$ & $<5$ & $\begin{array}{c}\text { Compris entre } 5 \\
\text { et 7,5 }\end{array}$ & $\begin{array}{c}>7,5 \text { et }< \\
10\end{array}$ & $>10$ \\
\hline
\end{tabular}

Tableau I : classes de tolérance de la norme

Une classe est ainsi attribuée aux différents modèles qualifiés conformément aux dispositions de la norme. Une fois la qualification terminée et validée, le fabricant peut indiquer sur son disposition «conforme à la norme E 18-100», ainsi que sa classification

S'ajoute aux essais d'exactitude, d'autres essais de fonctionnement :

- Des essais de facteurs d'influence pour les dispositifs électroniques (chocs, vibrations, Compatibilité électro magnétique, étanchéité IP...)

- Des essais de facteurs perturbateurs pour les dispositifs chimiques ou microbiologiques (influence de l'éclairement par exemple)

Les essais réalisés sont souvent destructifs impliquant donc la nécessité d'avoir une approche de qualification par lot c'est-à-dire par échantillonnage de la production. La norme prévoit ainsi des essais de type avec un échantillonnage minimal (10 individus) en vue de valider la conception.

4.2 - Application de la norme E 18-100 à des indicateurs / intégrateurs biologiques
Le Cemafroid a mis en cuvre la norme E 18-100 pour le compte d'un fabricant d'indicateur intégrateur biologique. Conformément à la norme, les essais doivent être réalisés par un laboratoire qui respecte les conditions préconisées dans le référentiel de bonnes pratiques BP X 10-042 et les prescriptions de la norme NF EN ISO 17025 . Les conditions atmosphériques ainsi que les conditions d'éclairage de référence y sont définis. Les enceintes thermostatiques utilisées doivent être caractérisées suivant la norme NF X 15140 ou la norme CEI 60 068-3-11 en charge. En régime établi, la valeur absolue de l'écart entre la température désirée et la température de chaque point de l'espace de travail est inférieure à ou égale à $1^{\circ} \mathrm{C}$. La stabilité maximale des températures dans l'espace de travail est inférieure de $2^{\circ} \mathrm{C}$. La chaine de mesure de température doit avoir une incertitude élargie $(\mathrm{k}=2)$ inférieure ou égale à $0,3^{\circ} \mathrm{C}$ entre $-20^{\circ} \mathrm{C}$ et $+40^{\circ} \mathrm{C}$. Les opérateurs réalisant les essais doivent être qualifiés pour l'analyse visuelle des dispositifs selon les préconisations du référentiel de Bonnes pratiques BP X 10-042, l'effet opérateur sur la mesure du temps doit être quantifié par le laboratoire.

Les essais ont été réalisés sur des ITT de marque « TopCryo » de la société Cryolog. Le «TopCryo» est un intégrateur temps/température (ITT) microbiologique sous forme d'étiquette autocollante. Il permet d'interpréter l'impact du temps et de la température sur la conservation des produits frais et ultra frais par un changement de couleur visible et irréversible : de vert à rouge. Son paramétrage biologique est défini selon le cahier des charges temps/température de chaque utilisateur et produits.

Une large gamme d'intégrateurs allant de quelques heures jusqu'à 12 jours a été développée pour couvrir des températures comprises entre $+2^{\circ} \mathrm{C}$ et $+12^{\circ} \mathrm{C}$ (incluant donc le domaine $+2^{\circ} \mathrm{C}-+8^{\circ} \mathrm{C}$ ) tout en intégrant les possibles ruptures de la chaine du froid à température ambiante. Les essais ont été effectués sur :

- Un lot de 10 dispositifs de l'extrémité minimale de la gamme, soit le modèle TOP B à $+2^{\circ} \mathrm{C}$ avec une EMT de $\pm 0,5^{\circ} \mathrm{C}$,

- Un lot de 10 dispositifs de l'extrémité maximale de la gamme, soit le modèle TOP I à $+12^{\circ} \mathrm{C}$ avec une EMT de $\pm 0,5^{\circ} \mathrm{C}$,

- Un lot de 10 dispositifs de milieu de gamme, soit le modèle TOP E à $+8^{\circ} \mathrm{C}$ avec une EMT de $\pm 0,5^{\circ} \mathrm{C}$.

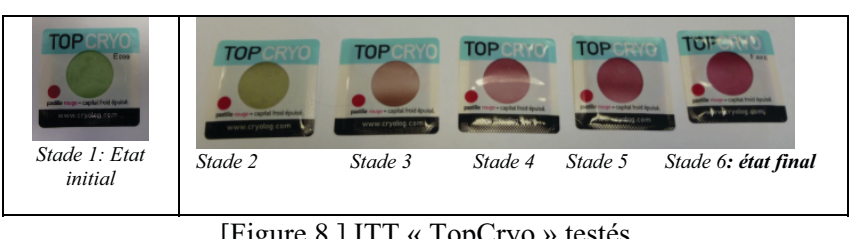

\section{3 - Résultats}

\section{Essais à températures constantes}

L'essai se déroule comme suit :

- Stabilisation de l'enceinte,

- Positionnement les dispositifs, soit 10 intégrateurs temps/température par modèles testés, dans l'enceinte thermostatique à la température d'essai, 
- Prise d'un cliché photographique toutes les 30 minutes • sur la durée de l'essai afin de visualiser les différents changements d'état,

- Acquisition de la température dans l'enceinte toutes les minutes et pendant toute la durée de l'essai.

\begin{tabular}{|c|c|c|}
\hline Profils & Durée $(\mathrm{h})$ & $\begin{array}{c}\text { Température } \\
\left({ }^{\circ} \mathrm{C}\right)\end{array}$ \\
\hline 1 & $\begin{array}{c}\text { Jusqu'à l'atteinte } \\
\text { de l'état final }\end{array}$ & Tréf \\
\hline 2 & $\begin{array}{c}\text { Jusqu'à l'atteinte } \\
\text { de l'état final }\end{array}$ & Tréf + EMT \\
\hline 3 & $\begin{array}{c}\text { Jusqu'à l'atteinte } \\
\text { de l'état final }\end{array}$ & Tréf - EMT \\
\hline 4 & $\begin{array}{c}\text { Jusqu'à l'atteinte } \\
\text { de l'état final }\end{array}$ & Tréf +2 EMT \\
\hline 5 & $\begin{array}{c}\text { Jusqu'à l'atteinte } \\
\text { de l'état final }\end{array}$ & Tréf -2 EMT \\
\hline
\end{tabular}

Les intégrateurs temps/température sont vérifiés à l'aide d'une source lumineuse de type F11 ( $18 \mathrm{~W}, 4000 \mathrm{~K}$, IRC 85), éclairage tube fluorescent industriel standard Europe- blanc/spectre à trois

\begin{tabular}{|c|c|c|c|c|c|c|}
\hline Profil & $\begin{array}{c}\mathbf{T} \\
\left({ }^{\circ} \mathbf{C}\right)\end{array}$ & $\begin{array}{c}\text { Durée } \\
\text { déclaré } \\
\mathbf{e}(\mathbf{h})\end{array}$ & $\begin{array}{c}\text { Durée } \\
\mathbf{m a x} \\
\mathbf{m} \text { murées } \\
\mathbf{( h )}\end{array}$ & $\begin{array}{c}\text { Erreur* } \\
\text { absolue }\end{array}$ & $\begin{array}{c}\text { Erreur } \\
* *(\%)\end{array}$ & Classe \\
\hline $\mathbf{1}$ & $+8,0$ & 51,3 & 53 & 1,7 & 3,21 & $\mathrm{~A}$ \\
\hline $\mathbf{2}$ & $+8,5$ & 46,5 & 48,5 & 2 & 4,12 & $\mathrm{~A}$ \\
\hline $\mathbf{3}$ & $+7,5$ & 54,3 & 57 & 2,7 & 4,74 & $\mathrm{~A}$ \\
\hline $\mathbf{4}$ & $+9,0$ & 44,1 & 44,5 & 0,4 & 0,90 & $\mathrm{~A}$ \\
\hline $\mathbf{5}$ & $+7,0$ & 59,2 & 60,5 & 1,3 & 2,15 & $\mathrm{~A}$ \\
\hline
\end{tabular}

bandes (raies) étroites.

Les résultats pour le TOP pour le modèle E sont les suivants :

*Erreur $=$ abs (valeur théorique annoncée CRYOLOG - valeur référence CEMAFROID)

**Erreur en $\%=($ Erreur en absolue* 100$) /$ valeur référence CEMAFROID.

Tableau III : Résultats d'essais statiques

Essais sur un profil de température dynamique:

Cet essai permet de mesurer la durée totale réelle du dispositif sur un profil de température dynamique. L'essai se déroule comme suit :

- Stabilisation de l'enceinte,

- Positionnement du dispositif, soit 10 intégrateurs temps/température par modèles testés dans l'enceinte thermostatique à la température de l'essai,

- Lancement du profil à réaliser dès la fermeture de l'enceinte thermostatique,

- Prise d'un cliché photographique toutes les 30 minutes sur la durée de l'essai afin de visualiser les différents changements d'état,

- Acquisition de la température dans l'enceinte toutes les minutes et pendant toute la durée de l'essai.
Le profil de températures dynamique est défini comme suit, dans la limite de Tmin et Tmax.

\begin{tabular}{|c|c|c|}
\hline Segment & $\begin{array}{c}\text { En \% de la durée totale } \\
\text { théorique à Tréf }\end{array}$ & $\begin{array}{c}\text { Température } \\
\left({ }^{\circ} \mathbf{C}\right)\end{array}$ \\
\hline $\mathbf{1}$ & $26 \%$ & Tref $\mathrm{x} 0,5$ \\
\hline $\mathbf{2}$ & $5 \%$ & Tref $\times 3$ \\
\hline $\mathbf{3}$ & $20 \%$ & Tref \\
\hline $\mathbf{4}$ & $2 \%$ & Tref $\times 5$ \\
\hline $\mathbf{5}$ & $16 \%$ & Tref \\
\hline $\mathbf{6}$ & $31 \%$ & Tref $\mathrm{x} 1,5$ \\
\hline
\end{tabular}

Tableau IV : essais dynamiques

Les intégrateurs temps/température sont vérifiés à l'aide d'une source lumineuse de type F11 (18 W, $4000 \mathrm{~K}$, IRC 85), éclairage tube fluorescent industriel standard Europe- blanc/spectre à trois bandes (raies) étroites.

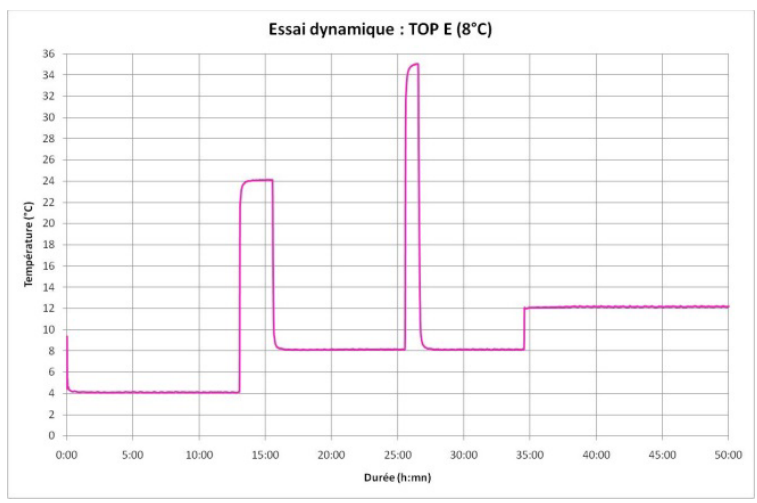

[Figure 9.] Température ambiante enregistrée dans l'enceinte, lors d'un essai sur un profil de température dynamique.

Résultats pour TOP E :

\begin{tabular}{|c|c|c|c|c|c|}
\hline TOP & $\begin{array}{c}\text { Durée } \\
\text { Cryolog } \\
(\mathrm{h})\end{array}$ & $\begin{array}{c}\text { Durée } \\
\text { Cemafroid } \\
(\mathrm{h})\end{array}$ & $\begin{array}{c}\text { Erreur* } \\
\text { en } \\
\text { absolue }\end{array}$ & $\begin{array}{c}\text { Erreur } \\
(\%)\end{array}$ & Classe \\
\hline $\begin{array}{c}\text { TOP } \\
\mathrm{E} \\
\left(8^{\circ} \mathrm{C}\right)\end{array}$ & 43,6 & 44,0 & 0,4 & 0,91 & $\mathrm{~A}$ \\
\hline
\end{tabular}

Tableau $\mathrm{V}$ : résultats des essais dynamiques

Les essais réalisés sur les IIT Top Cryo ont montré la pertinence du protocole d'essai de la norme NF 18100 et son applicabilité. Tous les essais préconisés ont pu être réalisés. Les ITT étaient dans le cas présent conformes aux performances annoncées par le fabricant, conforme à la classe $\mathrm{A}$ et les facteurs d'influence testés par ailleurs n'ont pas montré d'impact.

\section{Conclusion}

Si l'usage des IT et ITT pouvait être très utiles dans une stratégie de surveillance de la chaine du froid des produits de santé, il subsistait jusqu'à présent un handicap au développement de cette technique du fait qu'aucune norme ne définissait des modalités de justification des caractéristiques métrologiques de ces IT et ITT. La norme E 18100 comble cette lacune et permet désormais de disposer d'une méthode reproductible et métrologiquement robuste pour établir la traçabilité des seuils temps températures détectés pour un dispositif donné.

Les essais étant par nature réalisé sur les échantillons donnés, il convient d'appliquer cette norme dans le cadre d'une procédure 
d'évaluation bien plus large qui permet de garantir l'homogénéité de la production de ces indicateurs. La vérification périodique des lots dans le cadre d'une marque de certification de produit ou d'un label permettrait d'assoir et d'objectiver la démarche. C'est ce qu'entend proposer le Cemafroid dans le cadre du label Certicold Pharma à tous les constructeurs qui en feraient la demande. 